\title{
Optimal Sensor Location for Detecting Changes in Dynamical Behavior
}

\author{
MICHËLE BASSEVILLE, ALBERT BENVENISTE, MEMBER, IEEE, GEORGES V. MOUSTAKIDES, MEMBER, IEEE, AND
} ANNE ROUGÉE

\begin{abstract}
We address the problem of optimal sensor location for monitoring the eigenstructure of a multivariable dynamical system. The criterions which are optimized are the power of new tests designed for detecting and diagnosing changes in the eigencharacteristics of a system [3], [12]. The key points are the choice of the parameterization for computing the criterion and the comparison of designs with a different number of sensors. The discussion of the numerical results for sensor location includes the analysis of the effect of the geometry of the unknown excitation.
\end{abstract}

\section{INTRODUCTION}

$\mathrm{T}$ HE problem of optimal sensor location is of crucial importance in system design. In order to reduce the cost of instrumentation and increase the efficiency of the identifiers, state estimators or detectors are implemented for monitoring the system. Except for distributed parameter systems, this problem seems to have received little attention in the literature. Furthermore, it has been addressed more in view of good parameter estimation or state reconstruction, than for optimum monitoring of the system parameters and for change or failure detection.

\section{A. Short Review of Existing Results}

The results which are available so far, up to our knowledge, may be classified as follows.

1) Optimal Sensor Location for State Reconstruction: The problem is to find a measurement matrix $H$ which optimizes a criterion reflecting the performances of the optimum state estimator (or smoother) for the considered linear dynamical system. Various criterions have been investigated. Using a direct sensitivity analysis of the state estimate covariance matrix $P,[1]$ suggested to solve the nonlinear programming problem

$$
\min _{H} \operatorname{tr}(W P)
$$

where $W$ is a weighting matrix.

Several other authors [4], [7], [8] used indirect measures of performance $\Gamma$, such as Fisher information matrix (for state estimation) or observability matrix. Furthermore, several scalar performance indexes related to $\Gamma$ were studied. The most general one is [8], [7]

$$
m_{s}=\left(\frac{1}{n} \operatorname{tr} \Gamma^{s}\right)^{1 / s}, \quad s \leqq 0, n=\text { state dimension }
$$

Manuscript received February 18, 1986; revised April 22, 1987. Paper recommended by Associate Editor, R. C. Montgomery. This work was supported by IFREMER under Grant $84 / 7392$ and by CNRS GRECO SARTA.

M. Basseville is with IRISA/CNRS, 35042 Rennes Cedex, France.

A. Benveniste and G. V. Moustakides are with IRISA/INRIA, 35042 Rennes Cedex, France.

A. Rougée is with IRISA, 35042 Rennes Cedex, France.

IEEE Log Number 8716817. which, in some special cases, reduces to the following widely used criterions:

$$
\begin{array}{ll}
\lim _{s \rightarrow 0} m_{s}=|\Gamma|^{1 / n} & \text { (determinant norm) } \\
m_{-1}=n / \operatorname{tr}\left(\Gamma^{-1}\right) & \text { (trace norm) } \\
m_{-\infty}=\lambda_{\min }(\Gamma) & \text { (extremal eigenvalue). }
\end{array}
$$

Finally, the case of nonlinear systems was studied in [5], with the aid of the trace norm for the Fisher information matrix of the unknown parameters to be estimated.

One common feature of all these investigations is that the measurement matrix $H$ is supposed to be of "continuous" type, namely to have real (and not integer) coefficients.

The dual problem of optimum controller location was investigated in [8], with the aid of the above-mentioned general norm for the controllability matrix.

The problem of optimal sensor location for state estimation is closely related to the problem of optimal sensor location for control of systems, which has been extensively studied, especially for large space structures. Some significant references are [16][18].

2) Optimum Sensor and Controller Location for Distributed Parameter Systems:

These problems have been widely investigated for the last 15 years, as can be seen from the survey paper [6]. Sensor location for state estimation was studied, for example, in [10], using the trace norm of the error covariance of the optimum filter. The sensor location problem for identification of unknown parameters was solved in [9] with the aid of the determinant of the Fisher information matrix, and with application to a bubble-column loop bioreactor. Many other studies concerning these two types of problems are reported in [6], together with the solutions given to the problem of optimum controller and sensor location for optimum control of a distributed parameter system.

3) Optimal Sensor Location for Parameter Identification: Up to our knowledge, the only study made from that point of view is reported in [13], and is concerned with structural identification for both linear and nonlinear systems. The proposed solution is based upon an optimization of the trace norm of the Fisher information matrix. It is important to note that, in this case, the measurement matrix $H$ is a selection matrix (with coefficients equal to 0 or 1 ), and that the optimization is done by exhaustive search.

4) Optimal Sensor Location for Failure Detection: This problem is investigated in [15] in the framework of nonlinear systems. A reduced-order time-varying linear observer is designed for full state estimation in such a system. Inspection of the state estimates and/or several observer residuals leads to detection and diagnosis of the faults, without any statistical test. The optimum sensors location problem is then solved by exhaustive search for minimizing the observation cost associated to each set of measurements which is convenient for this fault detection strategy. 
This problem, together with the dual problem of optimum actuator location, is considered in [14] for large space structures. A degree of controllability (respectively, observability) which accounts for possible component failures, is defined and optimized over the admissible set of actuator (respectively, sensor) locations, either by exhaustive search or by solving an integer programming problem.

\section{B. Our Approach: Optimum Sensor Location for Change Detection}

In this paper, we address the problem of optimal sensor location for detecting changes in the eigenstructure of a dynamical system. This problem may be thought to belong to the same class as problem 4) in the sense that they both imply a change in the model which describes the system. Because of our detection and identification approaches, we actually also address problem 3 ). The underlying application is vibration monitoring for offshore platforms; the interested reader is referred to [3] for a presentation of this application. We derived statistical instrumental tests for detection and diagnosis of changes in the vibrating characteristics of a structure subject to an unknown nonstationary excitation. The numerical performances of these tests are reported in [2] for scalar signals and [3] for multivariable systems. The theoretical properties of these tests are investigated in [12] under stationarity assumptions: the criterion which is used for evaluating the performances of the tests is the classical detection power for a fixed level (false alarm rate).

The purpose of this paper is the investigation of the possible uses of such a type of criterion for designing optimal numbers and locations of sensors. We especially emphasize the key points of choice of parameterization for optimization in Section II, comparison of designs with different number of sensors in Section III, and influence of the geometry of the excitation upon the optimal design in Section IV. Numerical results obtained on a simulated structure are also reported in Section IV. Conclusions are given in Section V.

\section{Problem Statement-Choice of Parameterization}

We consider a dynamical system described by the following discrete-time state-space representation:

$$
\left\{\begin{array}{l}
X_{t+1}=F X_{t}+V_{t+1} \\
Y_{t}=H X_{t}
\end{array}\right.
$$

where the state $X$ is of dimension $n$, the observation $Y$ is of dimension $r \ll n$, and where $V_{t}$ is a Gaussian white noise with covariance matrix $Q$. The observation matrix $H$ is a selection matrix, i.e., we observe a limited number of state variables. The change or failure detection problem we solved in [3] is as follows: given a measurement matrix $H$, detect and diagnose changes in the eigenstructure of the system, i.e., the eigenvalues and the observed components of the eigenvectors of $F$, without knowing or using any estimate of the noise covariance matrix $Q$ (which is furthermore time-varying in [3]).

\section{A. New Test for Change Detection and Diagnosis}

For this purpose, we derived new statistical tests which may be summarized in the following way. The multivariable process (1) may be equivalently represented by the ARMA model:

$$
Y_{t}=\sum_{i=1}^{p} A_{i} Y_{t-i}+\sum_{j=0}^{p-1} B_{j} E_{t-j}
$$

where $\left(E_{t}\right)$ is a standard white noise. One possible way is to solve the following linear system of equations:

$$
\sum_{i=0}^{p} A_{i} H F^{p-i}=0
$$

with $A_{0}=-I_{r}$.

In such a case, the change detection problem is to detect changes in the AR parameters $A_{i}$ of (2), while considering the MA parameters $B_{j}$ as nuisance parameters; furthermore, deciding which poles and corresponding eigenvectors have changed would solve the diagnosis problem. We use a model validation approach. Given a nominal AR model $\theta^{0}$, where

$$
\theta^{T}=\left(A_{p}, \cdots, A_{1}\right)
$$

or a nominal observable model $\left(H_{0}, F_{0}\right)$, and a sample of observations $Y_{1}, \cdots, Y_{s}$, we consider what we call the instrumental statistics

$$
U_{N}(s)=\frac{1}{\sqrt{s}} \sum_{t=1}^{s} Z_{t}^{N} W_{t}^{T}
$$

where

$$
\begin{gathered}
Z_{t}^{N T}=\left(Y_{t-p}^{T}, \cdots, \begin{array}{r}
\left.Y_{t-p-N+1}^{T}\right) \text { is the vector of instruments } \\
W_{t}=Y_{t}-\theta^{\circ} T_{\phi_{t}}
\end{array}\right. \\
\phi_{t}^{T}=\left(Y_{t-p}^{T}, \cdots, Y_{t-1}^{T}\right) .
\end{gathered}
$$

We also introduce the corresponding vectors

$$
\begin{gathered}
\theta \stackrel{\triangleq}{=} \operatorname{col}\left(\theta^{T}\right) \\
\mathcal{U}_{N}(s) \triangleq \operatorname{col}\left(U_{N}(s)\right) \\
\triangleq \frac{1}{\sqrt{s}} \sum_{t=1}^{s} Z_{t}^{N} \otimes W_{t} \text { (Kronecker product) } .
\end{gathered}
$$

1) Detection: Our approach is based upon the following remark. If the nominal model $\theta^{0}$ still matches the new observations $Y_{\mathrm{I}}, \cdots, Y_{s}$, then the two vectors $Z_{\mathrm{l}}^{N}$ and $W_{l}$ in (5) are uncorrelated, which is no longer the case if a change occurred. This remark has been exploited in [3] according to the following lines.

The two hypotheses to be tested are:

$$
\begin{array}{ll}
\mathrm{f}_{0}: \theta=\theta^{\circ} & \text { no change } \\
\}_{3}: \theta=\theta^{\circ}+\frac{\delta \theta}{\sqrt{s}} & \begin{array}{l}
\text { small change in direction } \delta \theta, \\
\text { i.e., local alternative. }
\end{array}
\end{array}
$$

It may be shown [3] that, under $\mathrm{H}_{0}, U_{N}(s)(5)$ is zero-mean, and that, under $\mathrm{t}_{1}$, we have

$$
\xi_{1}\left(U_{N}(s)\right) \cong \mathcal{H}_{\rho, N}^{T} \delta \theta
$$

where

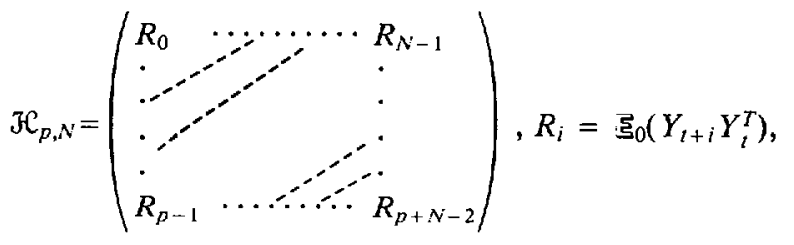

is the Hankel matrix of the process (2) under $\mathrm{i}_{0}$.

We assume that this Hankel matrix may be factorized as

$$
\mathcal{H}_{p, N}=\mathcal{O}_{p}\left(H_{0}, F_{0}\right) \mathcal{C}_{N}\left(F_{0}, G_{0}\right)
$$


where $\mathcal{O}_{p}$ is the observability matrix, $\mathcal{C}_{N}$ is the controllability matrix of system (1), and $G_{0}$ is the cross-correlation between $X$ and $Y$ under $\mathscr{H}_{0}$.

Because of (7) and (8), using $U_{N}(s)$, we are able to detect all the changes $\delta \theta$ except those satisfying $\delta \theta^{T} . \hat{\theta}_{p}\left(H_{0}, F_{0}\right)=0$.

But, as shown in [3], it turns out that, under assumption (8), all these changes $\delta \theta$ are precisely all the changes in the minimal representation of the system (1), which are the only changes of interest.

Furthermore, we also use the following local asymptotic normalities:

$$
\begin{gathered}
\text { under }[\mathrm{r}]_{0}, \mathcal{U}_{N}(s) \underset{s \rightarrow \infty}{\stackrel{\mathfrak{L}}{\longrightarrow}} \mathfrak{T}\left(0, \Sigma_{N}\right) \\
\text { under } \mathfrak{H}_{1}, \mathcal{U}_{N}(s) \underset{s \rightarrow \infty}{\stackrel{\mathcal{L}}{\longrightarrow}} \mathfrak{T}\left(\left(\mathcal{H}_{p, N}^{T} \otimes I_{r}\right) \delta \Theta, \Sigma_{N}\right)
\end{gathered}
$$

where

$$
\Sigma_{N}=\sum_{i=1-p}^{p-1} \Xi_{0}\left(Z_{t}^{N} Z_{t-i}^{N T} \otimes W_{t} W_{t-i}^{T}\right)
$$

is the covariance matrix of $\mathfrak{U}_{N}$.

We have thus transformed the initial change detection problem into a problem of detection of a change in the mean of a Gaussian process. As discussed in [3], the convenient test is then

$$
t_{0}(s)=\mathcal{U}_{N}^{T}(s) \Sigma_{N}^{-1} M\left(M^{T} \Sigma_{N}^{-1} M\right)^{-1} M^{T} \Sigma_{N}^{-1} \mathcal{u}_{N}(s)
$$

where

$$
M=\mathfrak{H C}_{p, N}^{T} D^{T} \otimes I_{r}
$$

and $D$ is such that $M$ is of full column rank $n r$. We refer the interested reader to [3] for details concerning the implementation of the global test (9) in the (real) situation where the noise covariance matrix $Q$ in (1)-or equivalently the MA parameters $B_{j}$ in (2)-are time-varying.

2) Diagnosis: For solving the diagnosis problem, namely deciding which eigenvalues and eigenvectors-of $F$ in (1) or equivalently of the AR part in (2)-have changed, our approach is the following. We still use the instrumental statistics $\mathcal{U}_{N}(6),(5)$, together with a relationship between changes in the eigencharacteristics of the system and changes in the AR parameters $\theta$. As we look for small changes, we use first-order Taylor expansions for $\theta$. Let $\phi$ be a minimal parameterization of the AR part of the process, for example, the modal characteristics, namely the eigenvalues $\mu$ of $F$ and the observed part $H \psi$ of the corresponding eigenvectors. Assume that $\phi \rightarrow \theta=f(\phi)$ is continuously differentiable in the neighborhood of the nominal model $\phi_{0}$. For monitoring a particular subset $\beta$ of the coordinates of $\phi$, we use the test given by formula (9) with

$$
M=\left(\mathcal{K}_{p, N}^{T} D^{T} \otimes I_{r}\right) \mathfrak{J}
$$

where $\mathcal{J}$ is the matrix obtained by selecting the convenient columns of the Jacobian matrix $f^{\prime}\left(\phi_{0}\right)$. For example, it may be shown [3] that writing (3) in the modal basis and differentiating it results in the following relationship:

$$
\begin{aligned}
\left(\Theta_{p}^{* T} \otimes I_{r}\right) d \Theta=\left(\begin{array}{rr}
J_{\psi} & i J_{\psi} \\
\bar{J}_{\psi} & -i \bar{J}_{\psi}
\end{array}\right) & \boldsymbol{H}\left(\begin{array}{c}
d \psi^{\mathrm{re}} \\
d \psi^{\mathrm{im}}
\end{array}\right) \\
& +\left(\begin{array}{l}
J_{\lambda} \\
\bar{J}_{\lambda}
\end{array}\right) d c+\left(\begin{array}{r}
i J_{\lambda} \\
-i \bar{J}_{\lambda}
\end{array}\right) d \omega
\end{aligned}
$$

where $\mathcal{O}_{p}^{*}$ is the observability matrix of the system (1) in the modal basis

$$
J_{\zeta}=\left(\begin{array}{ccc}
A\left(\mu_{1}\right) & 0 \\
0 & \cdots & 0\left(\mu_{l}\right)
\end{array}\right)
$$

$$
\begin{gathered}
J_{\psi}=\left(\begin{array}{cc}
\mu_{1} A^{\prime}\left(\mu_{1}\right) H \psi_{1} & 0 \\
0 & \cdots, A_{l} A^{\prime}\left(\mu_{l}\right) H \psi_{l}
\end{array}\right) \\
H=\left(\begin{array}{cc}
I_{l} \otimes H & 0 \\
0 & I_{l} \otimes H
\end{array}\right) \\
\mu_{j}=e^{\lambda_{j}}\left(\begin{array}{c}
1 \leqq j \leqq l=\frac{n}{2}
\end{array}\right) \\
\lambda_{j}=c_{j}+i \omega_{j}\left(\begin{array}{c}
c_{j}: \text { damping coefficient; } \\
\omega_{j}: \text { vibrating pulsation }
\end{array}\right) \\
A(X)=-\sum_{j=0}^{p} A_{j} X^{p-j} ; A^{\prime}(X)=-\sum_{j=0}^{p-1}(p-j) A_{j} X^{p-j-1} \\
\psi^{\text {re }} \text { and } \psi^{\text {im }} \text { are the real and imaginary } \\
\text { parts of the eigenvector } \psi
\end{gathered}
$$

For monitoring the subset $\beta$, we select columns in the $J$ matrices of the right-hand side of (12) in order to build a full column rank matrix $J_{\beta}$ such that

$$
\left(\mathcal{O}_{p}^{*} r \otimes I_{r}\right) d \Theta=J_{\beta} d \beta
$$

and then we use the test (9) together with $M$ defined by

$$
M=\left(\mathcal{C}_{N}^{* T} \otimes I_{r}\right) J_{\beta}
$$

where $\mathcal{C}_{\mathcal{N}}^{*}$ is the controllability matrix of the system (1) in the modal basis. Examples of such $J_{3}$ may be found in [3]. We call such type of tests "sensitivity tests." They will be of key importance for the sensor location problem, as will be seen in the next paragraph.

This approach for detection and diagnosis turns out to be very powerful, even for small changes in the eigencharacteristics. We emphasize that any change in the eigenstructure of the system can be detected with this method, even in the case of a loss of observability due to the change. Numerical results may be found in [2], [3]. A detailed theoretical analysis of the performances of these tests may be found in [12]. Some experimental results concerning the coupling effects between close modes are reported in [2].

\section{B. The Criterion to be Optimized}

We now discuss the problem of optimal sensor location with respect to the aim of detecting changes in the dynamics of the system (1): given a reference model $F_{0}$ in (1), how to choose the best measurement (selection) matrix $H$ in order to maximize the detection performances of the global test $t_{0}(9),(10)$ and/or the sensitivity tests (9), (11). As for investigating the theoretical properties of these tests $T$, the criterion we consider is the test's power $\beta$ for a fixed false alarm rate $\alpha$, which is a standard criterion in statistics. More precisely, we maximize

$$
\beta=\rho_{1}(T>\lambda)
$$

where the threshold $\lambda$ is chosen according to

$$
P_{0}(T>\lambda) \leqq \alpha
$$

Let us now emphasize that all the tests we have introduced have the following form. Let $U$ be a Gaussian random variable

$$
U \sim \Re(\mu, \Sigma)
$$


Assume first that

$$
\mu=M \nu
$$

is one parameterization of the mean value of $U$ such that $M$ is of full column rank $m$ and $\nu \in R^{m}$.

The test of $H_{0}: \mu=0$ against $H_{1}: \mu=M \nu$ is defined by

$$
T=U^{T} \Sigma^{-1} M\left(M^{T} \Sigma^{-1} M\right)^{-1} M^{T \Sigma^{-1}} U
$$

and is asymptotically distributed as a $\chi^{2}$ variable with $m$ degrees of freedom under both $H_{0}$ and $H_{1}$. Under $H_{0}, T$ is centered, but under $H_{1}$ the distribution of $T$ has a noncentrality parameter equal to

$$
\gamma^{2}={ }_{\nu}^{T} \Gamma \nu
$$

where

$$
\Gamma=M^{T} \Sigma^{-1} M .
$$

If we now assume that the rank of $M$ in (18) is $q<m$, let $D$ be the matrix containing the basis vectors of a complement of the kernel of $M$. Then

$$
\nu=D \xi+\nu_{*}
$$

where $\nu_{*} \in \operatorname{Ker}(M)$ and thus

$$
\mu=M \nu=M D \xi
$$

with $M D$ of full column rank $q$ and $\xi \in R^{q}$.

The test of $\mu=0$ against $\mu=M D \xi$ is

$$
U^{T} \Sigma^{-1} M D\left(D^{T} M^{T} \Sigma^{-1} M D\right)^{-1} D^{T} M^{T} \Sigma^{-1} U
$$

The corresponding noncentrality parameter is still (20) because $\xi^{T} D^{T} M^{T} \Sigma^{-1} M D \xi=\nu^{T} M^{T} \Sigma^{-1} M \nu$. In other words, whatever the rank of $M$ in (18) is, the noncentrality parameter is defined by (20).

Consequently, the threshold $\lambda$ depends only upon the number of degrees of freedom [which is equal to $n r$ for the global test (9) (10)], while the power $\beta$ is an increasing function of $\gamma(20)$, for a given $\lambda$. We will thus concentrate our efforts on the optimization, with respect to the measurement matrix $H$, of the quadratic form defined by $\Gamma\left(20^{\prime}\right)$.

1) Choice of Parameterization: In order to optimize the quadratic form defined by $\Gamma\left(20^{\prime}\right)$, we have to choose a scalar criterion. As many other authors (see Section I), we have chosen the trace norm and thus the following criterion:

$$
\begin{aligned}
C(M) & =\operatorname{Tr}(\Gamma) \\
& =\operatorname{Tr}\left(M^{T} \Sigma^{-1} M\right) .
\end{aligned}
$$

This choice may be justified using the following remark [8]:

$$
\int_{\substack{\nu \in R^{m} \\ T_{p=1}}} \nu^{T} M^{T} \Sigma^{-1} M \nu d \nu=\frac{C_{m}}{m} C(M)
$$

where $C_{m}$ is the area of the unit sphere in $R^{m}$. In other words, the criterion $C(M)$ is nothing but the mean value of the noncentrality parameter when $\nu$ covers the unit sphere in $R^{m}$.

The key point here is that the criterion $C(M)$ does depend upon the parameterization which is chosen for the mean value $\mu$ of the instrumental statistics $\mathcal{U}$ under $\mathrm{H}_{1}$, since $C(M P)$ is generally different from $C(M)$ even if $P$ is a change of basis. The first basic consequence, as far as optimal sensor location is concerned, is that it is necessary to choose a parameterization of the mean of $\mathcal{U}$ which does not depend upon the location of the sensors. From (3) we can see that the ARMA representation (2) depends upon the sensors location. Thus, we conclude that the criterion

$$
C_{N}=\operatorname{tr}\left(\Gamma_{N}\right)=\operatorname{Tr}\left[\left(\mathcal{K}_{p, N} \otimes I_{r}\right) \Sigma_{N}^{-1}\left(\mathcal{H C}_{p, N}^{T} \otimes I_{r}\right)\right]
$$

which was used in [12] for optimization over $N$ for fixed $H$, is no more convenient for optimizing over $H$.

From now on, we will thus consider parameterizations related to the characteristics of the system: eigen (or modal) parameters, state transition matrix $F$. Before doing that, we notice that the following invariance property of the criterion $C(21)$ :

$$
P P^{T}=I_{m} \Rightarrow C(M P)=C(M) \text {. }
$$

2) Several Possible Criterions: According to the previous discussions about the choice of parameterization for sensors location in 1) and, on the other hand, about diagnosis via sensitivity tests in A.2), it results that several criterions of the type (21) may be considered, using various Jacobian matrices $J_{B}$ in $M$ (15).

a) Global Modal Sensitivity: One subset of parameters which is of particular interest, as far as vibration monitoring is concerned, is the set of the modal parameters, namely the vibrating pulsations $\omega_{j}$ and the eigenvectors $\psi_{j}$. Of course, only the observed part $H \psi_{j}$ of the eigenvectors can be monitored, but we nevertheless choose the whole set $\left(\omega_{j}, \psi_{j}\right)$ to parameterize the system independently of the observation matrix $H$. We have chosen not to monitor the damping coefficients $c_{j}$ (real part of the eigenvalue $\lambda_{j}$ ) because they are usually not precisely identified. In this case, using (12), (15), and (7), the mean value of $\mathcal{u}_{N}$ under $\mathrm{H}_{1}$ has the following parameterization:

$$
\Xi_{\mathfrak{l}}\left(\mathcal{U}_{N}\right)=\left(\mathcal{C}_{N}^{* T} \otimes I_{r}\right) \mathcal{J} \mathcal{L}\left(\begin{array}{c}
d \psi^{\mathrm{re}} \\
d \psi^{\mathrm{im}} \\
d \omega
\end{array}\right)
$$

where

$$
\mathcal{L}=\left(\begin{array}{ccc}
I_{l} \otimes H & 0 & 0 \\
0 & I_{l} \otimes H & 0 \\
0 & 0 & I_{I}
\end{array}\right)
$$

and

$$
\mathfrak{d}=\left(\begin{array}{ccc}
J_{\psi} & i J_{\psi} & J_{\lambda} \\
\bar{J}_{\psi} & -i \bar{J}_{\psi} & \bar{J}_{\lambda}
\end{array}\right)
$$

with $J_{\psi}$ and $J_{\lambda}$ given in (13) and $2 l=n$.

Since $H$ is a selection matrix, we have

$$
\mathscr{L}^{T}=I \text {. }
$$

Thus, because of the discussion following formulas (20) and of (23), the global modal criterion is defined according to (21) as

$$
C_{\omega, \psi}=\operatorname{Tr}\left[\mathfrak{J}^{T}\left(\mathcal{C}_{N}^{*} \otimes I_{r}\right) \Sigma_{N}^{-1}\left(\mathcal{C}_{N}^{* T} \otimes I_{r}\right) \mathfrak{d}\right] .
$$

It is of interest to notice that, because $J_{\psi}$ and $J_{\lambda}$ are blockdiagonal, we have

$$
C_{\omega, \psi}=\sum_{j=1}^{l} C_{j}
$$

where $C_{j}$ is the sensitivity criterion corresponding to only one mode, i.e., one pulsation and one eigenvector.

Numerical results concerning the criterion (24) will be presented in Section IV. The optimization is done by exhaustive search.

b) Sensitivity w.r.t. the F Matrix: Using the same approach as in the previous paragraph, we now differentiate (3) to obtain a connection between variations in $\theta$ and variations in $F$. Thus, we 
get

$$
d \theta^{T} \cdot \mathcal{O}_{p}(H, F)=-\sum_{j=0}^{p-1} A_{j} H d\left(F^{p-j}\right)
$$

where $\mathcal{O}_{p}(H, F)$ is the observability matrix of system (1). Using

$$
d F^{k}=\sum_{i=0}^{k-1} F^{k-1-i} \cdot d F \cdot F^{i}
$$

and introducing the MA parameters

$$
B_{k}=H F^{k}-A_{\mathrm{l}} H F^{k-1}-\cdots A_{k} H
$$

we finally obtain

$$
d \theta^{T} \cdot \mathcal{O}_{p}(H, F)=\sum_{k=0}^{p-1} B_{p-k-1} \cdot d F \cdot F^{k}
$$

or equivalently

$$
\left(\Theta_{p}^{T}(H, F) \otimes I_{r}\right) d \Theta=J_{F} \operatorname{col}(d F)
$$

where

$$
J_{F}=\sum_{k=0}^{p-1}\left(F^{T k} \otimes B_{p-k-1}\right)
$$

Using the factorization (8) of the Hankel matrix, we get

$$
E_{1}\left(\mathcal{U}_{N}\right)=\left(\mathcal{C}_{N} \otimes I_{r}\right) J_{F} \cdot \operatorname{col}(d F) .
$$

Therefore, the sensitivity criterion with respect to the variations in $F$ is thus

$$
C_{F}=\operatorname{Tr}\left[J_{F}^{T}\left(\mathcal{C}_{N} \otimes I_{r}\right) \Sigma_{N}^{-1}\left(\mathfrak{C}_{N}^{T} \otimes I_{r}\right) J_{F}\right] .
$$

As $J$ in (24) and $J_{F}$ have, respectively, $(2 r+1) l$ and $(2 l)^{2}$ columns $(n=2 l)$, and because $r \ll l$, the criterion $C_{F}$ is much more computationally expensive than the criterion $C_{\epsilon^{\prime}, \psi}(24)$.

\section{COMParisons of Designs With Different Number of SENSORS}

According to the discussion presented in Section II-B, up to now we have basically been able to compare different sensor locations corresponding to the same number of sensors: recall that the criterion (21) is directly related to the test power $\beta(16)$, which is defined for a fixed threshold $\lambda$, and that $\lambda$ depends upon the number $q$ of degrees of freedom.

The purpose of this section is to define a correcting factor which allows the comparison between different number of sensors. We emphasize that we do not take into account the cost of an additional sensor. Our criterion only compares the detection performances of different designs.

First notice that the power $\beta(16)$ is computed as

$$
\beta=P\left(x_{1}^{2}+\cdots+x_{q}^{2} \geqq \lambda\right) \triangleq P\left(D_{\lambda}\right)
$$

where $x_{i}=z_{i}+\gamma_{i}$ and $\left(z_{i}\right)$ are zero mean independent identically distributed Gaussian variables, and $\gamma_{i}$ is the mean of $x_{i}$. Because of symmetry, we have

$$
P\left(\mathfrak{D}_{\lambda}\right)=P\left(x_{1}^{2}+\cdots+x_{q}^{2} \geqq \lambda\right)=P\left(\left(z_{1}+\gamma\right)^{2}+z_{2}^{2}+\cdots+z_{q}^{2} \geqq \lambda\right)
$$

where $\gamma^{2}=\gamma_{1}^{2}+\cdots+\gamma_{q}^{2}$ is the noncentrality parameter. Since we consider only small changes, i.e., $\gamma$ is small, we can keep only the first two nonzero terms of the Taylor expansion of (25)

$$
\begin{aligned}
& \beta=\int_{\mathfrak{D}_{\lambda}} \cdots \int \frac{1}{\sqrt{2 \pi}} e^{-1 / 2\left(x_{1}-\gamma\right)^{2}}\left(\prod_{i=2}^{q} \frac{e^{-x_{i}^{2} / 2}}{\sqrt{2 \pi}}\right) d x_{1} \cdots d x_{q} \\
& \cong \int_{\mathfrak{D}_{\lambda}} \cdots \int\left(\prod_{i=1}^{q} \frac{e^{-x_{i}^{2} / 2}}{\sqrt{2 \pi}}\right) d x_{1} \cdots d x_{q} \\
& +\gamma \int_{D_{\lambda}} \cdots \int x_{1} \frac{e^{-x_{1}^{2} / 2}}{\sqrt{2 \pi}}\left(\prod_{i=2}^{q} \frac{e^{-x_{i}^{2} / 2}}{\sqrt{2 \pi}}\right) d x_{1} \cdots d x_{q} \\
& +\frac{\gamma^{2}}{2} \int_{D_{\lambda}} \cdots \int\left(x_{1}^{2}-1\right)\left(\prod_{i=1}^{q} \frac{e^{-x_{i}^{2} / 2}}{\sqrt{2 \pi}}\right) d x_{1} \cdots d x_{q}+O\left(\gamma^{3}\right) \\
& =\alpha+\frac{\gamma^{2}}{2} \epsilon_{q}+0\left(\gamma^{3}\right)
\end{aligned}
$$

where

$$
\epsilon_{q}=\int_{D_{\lambda}} \cdots \int\left(x_{1}^{2}-1\right)\left(\prod_{i=1}^{q} \frac{e^{-x_{i}^{2} / 2}}{\sqrt{2 \pi}}\right) d x_{1} \cdots d x_{q} .
$$

But

$$
\begin{aligned}
\epsilon_{q} & =\int_{D_{\lambda}} \cdots \int x_{1}^{2}\left(\prod_{i=1}^{q} \frac{e^{-x_{i}^{2} / 2}}{\sqrt{2 \pi}}\right) d x_{1} \cdots d x_{q}-\alpha \\
& =\frac{1}{q} \int_{D_{\lambda}} \cdots \int\left(x_{1}^{2}+\cdots+x_{q}^{2}\right\}\left(\prod_{i=1}^{q} \frac{e^{-x_{i}^{2} / 2}}{\sqrt{2 \pi}}\right) d x_{1} \cdots d x_{q}-\alpha \\
& =\frac{1}{q}\left[{ }_{\mid l}\left[\chi_{q}^{2} \cdot 1_{\left\{x_{q}^{2} \geq \lambda\right\}}\right]-\alpha\right.
\end{aligned}
$$

where $\chi_{q}^{2}$ is a $\chi^{2}$ variable with $q$ degrees of freedom. Since $q$ is usually large, we may use the following Gaussian approximation:

$$
\frac{\chi_{q}^{2}-q}{\sqrt{q}} \sim \Re(0,1) .
$$

On the other hand, let $\phi$ be the Gaussian cumulative distribution function. If we define $\delta$ by

$$
\delta=\phi^{-1}(1-\alpha)
$$

then, because of (27), the threshold $\lambda$ in (17) is approximately

$$
\lambda \cong q+\delta \sqrt{q} \text {. }
$$

Thus

$$
\begin{aligned}
\vec{s}\left(\chi_{q}^{2} \cdot 1_{\left\{\chi_{q}^{2} \geqq \lambda\right\}}\right) & =\sqrt{q}\left[\underline{[}\left(\frac{\chi_{q}^{2}-q}{\sqrt{q}} 1_{\left\{\chi_{q}^{2} \geqq \lambda\right\}}\right)\right. \\
& \left.+\sqrt{q} E\left(1_{\left\{x_{q}^{2} \geqq \lambda\right\}}\right)\right] \\
& =\sqrt{q}\left[\int_{\delta}^{+\infty} x \frac{e^{-x^{2} / 2}}{\sqrt{2 \pi}} d x+\sqrt{q} \alpha\right] \\
& =\sqrt{q}\left(\frac{e^{-\delta^{2} / 2}}{\sqrt{2 \pi}}+\sqrt{q} \alpha\right) .
\end{aligned}
$$

Therefore

$$
\epsilon_{q}=\frac{e^{-\delta^{2} / 2}}{\sqrt{2 \pi q}}
$$




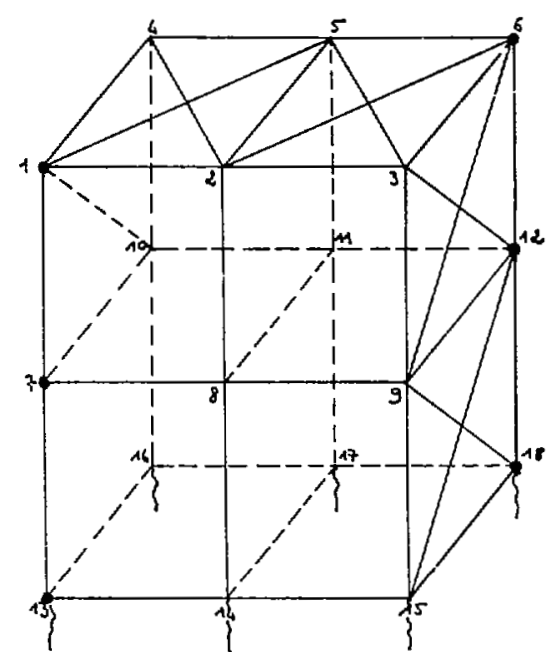

Fig. 1. Simulation system.

In other words, we have proved that

$$
\beta=\alpha+\frac{\gamma^{2}}{2} \frac{e^{-\delta^{2} / 2}}{\sqrt{2 \pi q}}+0\left(\gamma^{3}\right)
$$

Since $\delta$ (28) does not depend upon $q$, the convenient quantity to compare locations of different number of sensors is

$$
(\beta-\alpha) e^{\delta^{2} / 2}
$$

which is equal to

$$
\frac{\gamma^{2}}{2 \sqrt{2 \pi q}}
$$

up to second order.

Because of (20) and (22), integrating the two sides of this last equality leads to

$$
(\beta-\alpha) e^{\delta^{2} / 2}=\frac{1}{2 \sqrt{2 \pi q}} C(M)
$$

Therefore, the criterion (21) has to be divided by the square root of the number of degrees of freedom.

Numerical results involving this criterion will be presented in the next section.

\section{NUMERICAL Results}

In this section, we present numerical results concerning the criterion (24) computed for a simplified platform model. This simulation model is a nonsymmetric tied down system of 18 masses of one degree of freedom, connected by springs, as shown in Fig. 1, with known weights, stiffness and damping coefficients. No signals were generated; the theoretical values of the criterion were computed from the physical characteristics of the system and for a given excitation. Actually, it may be shown that everything in (24) can be computed as functions of $(H, F)$ and the theoretical covariances $R_{k}$ of the observation $Y$. We insist upon the fact that, even though the tests (9) may be computed without knowing the excitation $V$ in (1), our criterion does depend upon the excitation (through its covariance $Q$ ). This dependency is analytically complex, and thus will be studied only via numerical computations made with four different covariance matrices $Q$.

In order to mimic the effect of the swell, and assuming that the excitations at different points are independent, we selected diagonal covariance matrices $Q_{i}(i \leqq i \leqq 4)$; the diagonals are given in Table $I$, together with the values of the 18 masses. Excitation 1 is stronger than excitation 2 on the top level of the structure. Excitation 3 tries to simulate a dominant excitation on the "leg" 3-9-15. Excitation 4 acts in a similar way on the leg 612-18.

As we have previously mentioned, the optimization has been done by exhaustive search among a set of possible sensor locations. According to experiments currently performed on real offshore platforms, the locations which we have used correspond to the selection of $r=2,3$, or 4 , a total number of sensors located on each of the two opposite "legs" 1-7-13 and 6-12-18, with 1 or 2 sensors on each leg, resulting in a set of 36 possible locations.

The four global modal criterions $C_{i}(1 \leqq i \leqq 4)$ have been computed applying formula $\left(24^{\prime}\right)$, and then multiplied by the correcting factor $1 / \sqrt{m}$ according to (30). Here $m=36 r-18$, because we have chosen $p$ to satisfy $r p=n=36$ and $N=p$ and because we have omitted the damping coefficients $c_{j}$. Since the computations were done with the aid of the additive decomposition $\left(24^{\prime}\right)$, for each sensor location and each excitation, we display in fact 19 criterions, namely the 18 modal criterions $C_{j}$ which monitor separately the 18 eigenvectors and frequencies, plus their sum $C_{c, \dot{\psi}}$. Figs. 2-5 correspond to the excitations 1 to 4 , respectively. They are drawn with automatic scaling. The $x$-axis is devoted to the 36 sensor locations listed in the second block column of Table II, the $y$-axis to the 19 criterions, with the global model criterion on the back. The values of these four global criterions are listed in the first block column of Table II, and plotted in Fig. 6.

We first notice that the four "profiles" of Figs. 2-5, and the four curves of Fig. 6, are quite similar, showing a not too strong dependency of the criterions upon the excitation. Another way of checking this relative independency consists in sorting, for each excitation, the different locations according to the decreasing values of the global modal criterion. This is shown in the third block column of Table II. If the criterion $C_{\omega, 4}$ was independent upon the excitation, we would get the same rank value $\mathbb{R}_{i}$ on each row (i.e., for each location). We can see that the real situation is not dramatically different from this ideal situation.

Two other important facts may also be deduced from Table II. First, it does not seem really necessary to have 4 sensors for good detection, because good scores are obtained with 3 sensors conveniently located. Second, sensor locations which do not involve mass number 1 always get bad scores.

Finally, we checked another important point, from a numerical point of view. It turns out that the covariance matrix $\Sigma$, of size $36 r$, may be ill-conditioned for certain sensor locations [3]. Thus, it has been inverted using a singular value decomposition together with a test concerning the singular vectors: retain only the singular values which correspond to a pair of singular vectors $\left(U_{i}, V_{i}\right)$ such that

$$
U_{i}^{T} \cdot V_{i} \geqq \lambda \cong 1 .
$$

All the previously reported computations were made with $\lambda=$ 0.99 , which results in keeping very small singular values $(D-$ 15). A more severe choice $\lambda=0.999$ was tried in the case of excitation 2. The corresponding "profile" is in Fig. 7, and the comparison between the two global modal criterions corresponding to these two choices of $\lambda$ is made in Fig. 8. This effect seems to be negligible.

\section{Conclusion}

We have addressed the problem of optimal sensor location from the nonstandard point of view of failure detection with statistical 
TABLE I

THE 18 MASSES AND THE 4 EXCITATIONS

\begin{tabular}{c|l|l|l|l|}
\hline Masses & $Q_{1}$ & $Q_{2}$ & $Q_{3}$ & $Q_{4}$ \\
\hline 128 & 0.1 & 0.01 & 0.01 & 0.01 \\
64 & 0.1 & 0.01 & 0.03 & 0.01 \\
64 & 0.1 & 0.01 & 0.05 & 0.03 \\
64 & 0.1 & 0.01 & 0.01 & 0.01 \\
64 & 0.1 & 0.01 & 0.01 & 0.03 \\
64 & 0.1 & 0.01 & 0.03 & 0.05 \\
32 & 3 & 3 & 2 & 2 \\
32 & 3 & 3 & 3 & 2 \\
32 & 3 & 3 & 5 & 3 \\
32 & 3 & 3 & 2 & 2 \\
32 & 3 & 3 & 2 & 3 \\
32 & 3 & 3 & 3 & 5 \\
32 & 1 & 1 & 1 & 1 \\
32 & 1 & 1 & 1.2 & 1 \\
32 & 1 & 1 & 1.5 & 1.2 \\
32 & 1 & 1 & 1 & 1 \\
32 & 1 & 1 & 1 & 1.2 \\
32 & 1 & 1 & 1.2 & 1.5 \\
\hline- & & &
\end{tabular}

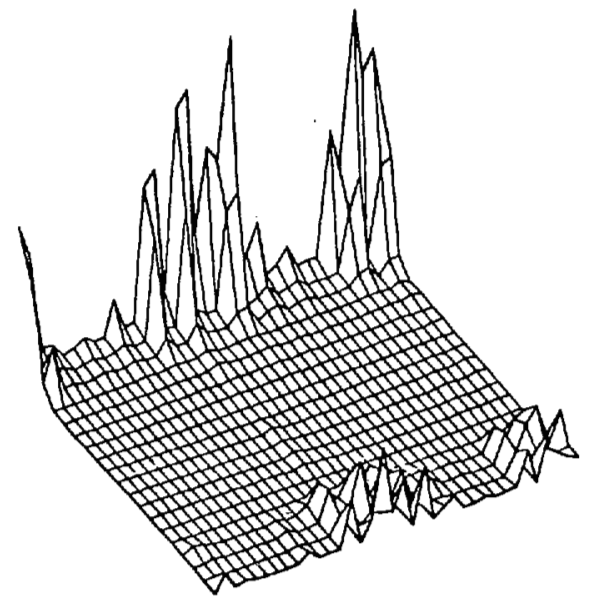

Fig. 2. Criterions for excitation 1.

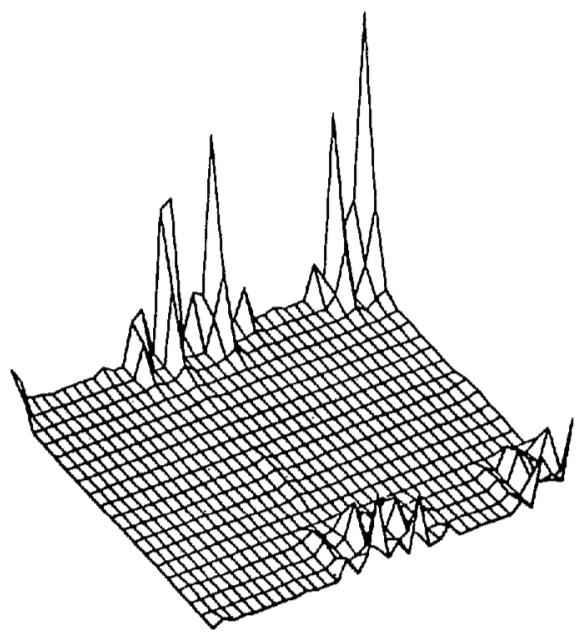

Fig. 3. Criterions for excitation 2.

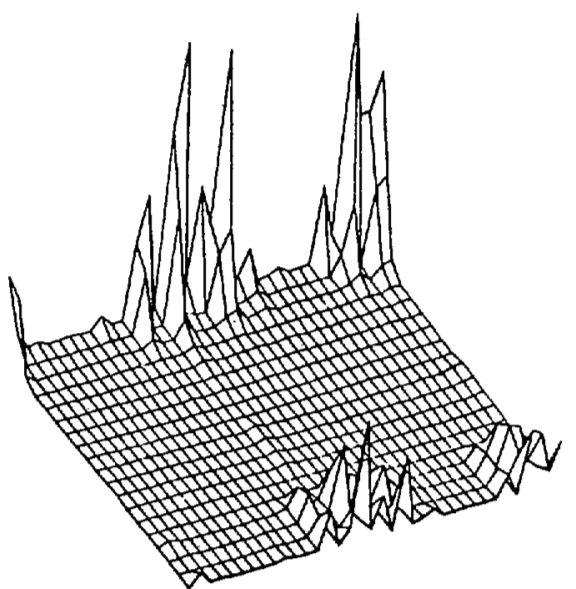

Fig. 4. Criterions for excitation 3.

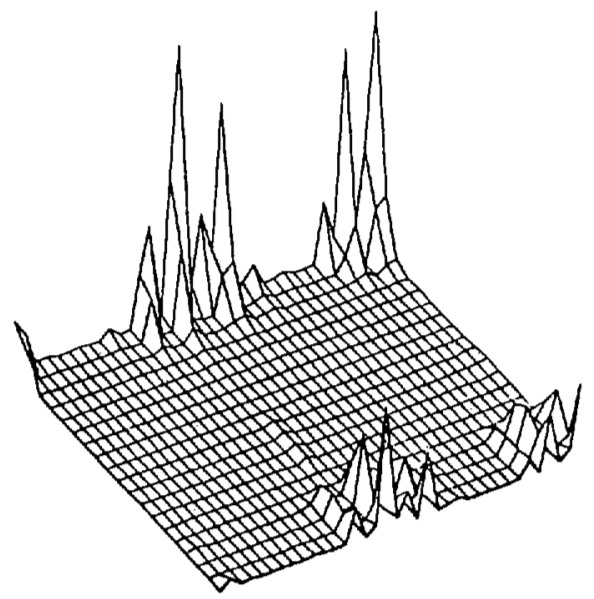

Fig. 5. Criterions for excitation 4. 
TABLE II

Global MOdal CRITERIONS, SENSOR Locations, AND RANKS OF THESE LOCATIONS

\begin{tabular}{|c|c|c|c|c|c|c|c|c|}
\hline $\mathrm{C}_{1}$ & $\mathrm{C}_{2}$ & $\Sigma_{3}$ & $\mathrm{C}_{4}$ & locations & $g_{1}$ & $\boldsymbol{x}_{2}$ & $\mathbb{Q}_{3}$ & $\boldsymbol{R}_{4}$ \\
\hline $\begin{array}{r}4584.95 \\
2779.70 \\
521.38 \\
494.86 \\
84.97 \\
193.86 \\
379.87 \\
171.37 \\
178.95 \\
1285.79 \\
252.77 \\
331.11 \\
4485.33 \\
4942.17 \\
920.08 \\
6710.24 \\
3181.27 \\
2516.45 \\
5075.12 \\
4583.91 \\
8492.11 \\
266.61 \\
1283.98 \\
2154.80 \\
326.28 \\
569.78 \\
1039.12 \\
299.39 \\
352.32 \\
325.54 \\
4356.40 \\
3047.30 \\
8115.30 \\
5915.87 \\
6621.73 \\
4730.57\end{array}$ & $\begin{array}{r}13927.54 \\
3336.14 \\
1318.50 \\
1117.72 \\
99.55 \\
209.18 \\
551.57 \\
178.93 \\
157.64 \\
2818.45 \\
249.27 \\
325.30 \\
15122.63 \\
18680.62 \\
1429.97 \\
50456.35 \\
52996.73 \\
4398.79 \\
17866.14 \\
15286.35 \\
69968.78 \\
263.04 \\
3021.52 \\
12752.83 \\
320.52 \\
716.74 \\
1680.20 \\
60.35 \\
362.35 \\
365.22 \\
12071.36 \\
5521.69 \\
62165.82 \\
21819.96 \\
29646.45 \\
92273.57\end{array}$ & $\begin{array}{r}12823.63 \\
3420.44 \\
602.08 \\
852.82 \\
104.14 \\
191.30 \\
389.20 \\
153.93 \\
176.06 \\
2092.91 \\
243.77 \\
295.10 \\
12444.48 \\
19595.70 \\
1213.67 \\
29588.83 \\
43887.75 \\
4071.46 \\
18607.38 \\
12424.14 \\
41037.34 \\
254.80 \\
2156.51 \\
6036.47 \\
296.12 \\
607.21 \\
1516.31 \\
261.89 \\
321.29 \\
345.22 \\
13079.95 \\
4549.94 \\
41943.61 \\
23793.90 \\
23836.61 \\
30480.11\end{array}$ & $\begin{array}{r}8979.35 \\
2641.92 \\
573.26 \\
743.86 \\
116.15 \\
185.49 \\
400.88 \\
154.98 \\
133.65 \\
1992.84 \\
249.40 \\
302.41 \\
9988.36 \\
17663.90 \\
1258.72 \\
25028.90 \\
46831.74 \\
5020.22 \\
16550.33 \\
9657.36 \\
34452.24 \\
240.94 \\
1881.52 \\
4525.13 \\
268.46 \\
556.33 \\
1257.38 \\
255.66 \\
350.20 \\
392.65 \\
10927.86 \\
4085.31 \\
36181.32 \\
2513.63 \\
17637.21 \\
41055.57\end{array}$ & 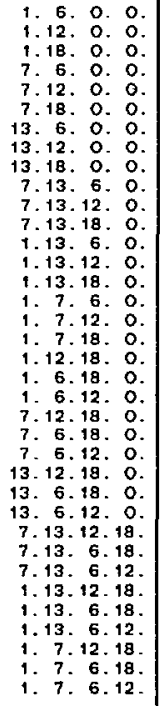 & $\begin{array}{r}10 . \\
15 . \\
23 . \\
24 . \\
36 . \\
33 . \\
25 . \\
35 . \\
34 . \\
18 . \\
32 . \\
27 . \\
12 . \\
8 . \\
21 . \\
4 . \\
3 . \\
16 . \\
7 . \\
11 . \\
1 . \\
31 . \\
19 . \\
17 . \\
28 . \\
22 . \\
20 . \\
30 . \\
26 . \\
29 . \\
13 . \\
14 . \\
2 . \\
6 . \\
5 . \\
9 .\end{array}$ & $\begin{array}{r}12 . \\
17 . \\
22 . \\
23 . \\
35 . \\
32 . \\
25 . \\
33 . \\
34 . \\
19 . \\
31 . \\
28 . \\
11 . \\
8 . \\
21 . \\
5 . \\
4 . \\
16 . \\
9 . \\
10 . \\
2 . \\
30 . \\
18 . \\
13 . \\
29 . \\
24 . \\
20 . \\
36 . \\
27 . \\
26 . \\
14 . \\
15 . \\
3 . \\
7 . \\
6 . \\
1 .\end{array}$ & $\begin{array}{r}11 . \\
17 . \\
24 . \\
22 . \\
36 . \\
33 . \\
25 . \\
35 . \\
34 . \\
19 . \\
32 . \\
29 . \\
12 . \\
8 . \\
21 . \\
5 . \\
1 . \\
16 . \\
9 . \\
13 . \\
3 . \\
31 . \\
18 . \\
14 . \\
28 . \\
23 . \\
20 . \\
30 . \\
27 . \\
26 . \\
10 . \\
15 . \\
2 . \\
7 . \\
6 . \\
4 .\end{array}$ & $\begin{array}{r}12 . \\
16 . \\
23 . \\
22 . \\
36 . \\
33 . \\
25 . \\
34 . \\
35 . \\
18 . \\
31 . \\
28 . \\
10 . \\
6 . \\
20 . \\
5 . \\
1 . \\
13 . \\
8 . \\
11 . \\
4 . \\
32 . \\
19 . \\
14 . \\
29 . \\
24 . \\
21 . \\
30 . \\
27 . \\
26 . \\
9 . \\
15 . \\
3 . \\
17 . \\
7 . \\
2 .\end{array}$ \\
\hline
\end{tabular}

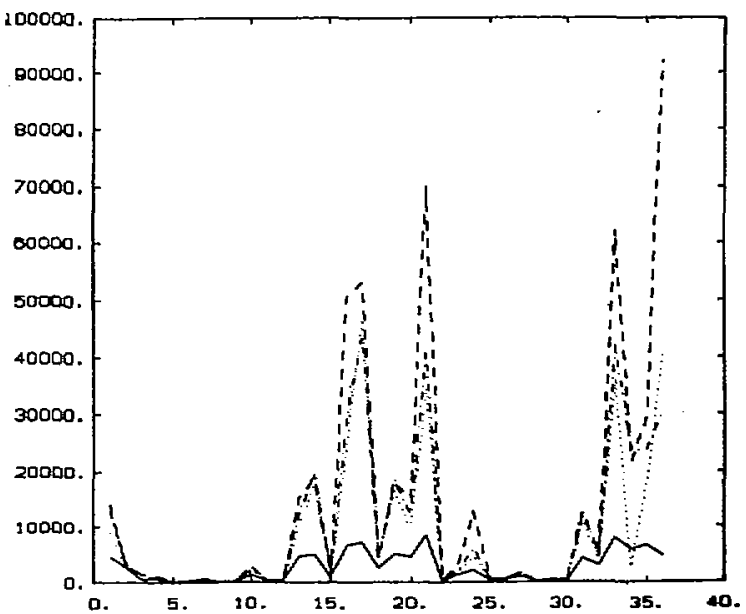

\section{excitation 1 excitation 2 excitation 3 excitation 4}

Fig. 6. The four global modal criterions.

tests. We have derived criterions based upon the power of the detection and diagnosis tests we recently developed for vibration monitoring [3]. Several key points have been discussed and solved, namely: choice of parameterization for optimization of sensor location, comparison of designs with different number of sensors, and influence of the excitation.

Further investigations should include a deeper understanding of the adequacy of the mean criterion (22); actually, we compute the mean power of our test for detecting any type of change with unit "magnitude." It turns out that some locations, corresponding nearly to local minima of the criterion, actually are more convenient (than optimal ones) for detecting some types of physical changes. Our opinion is that a more convenient criterion could be obtained using Jacobians with respect to physical

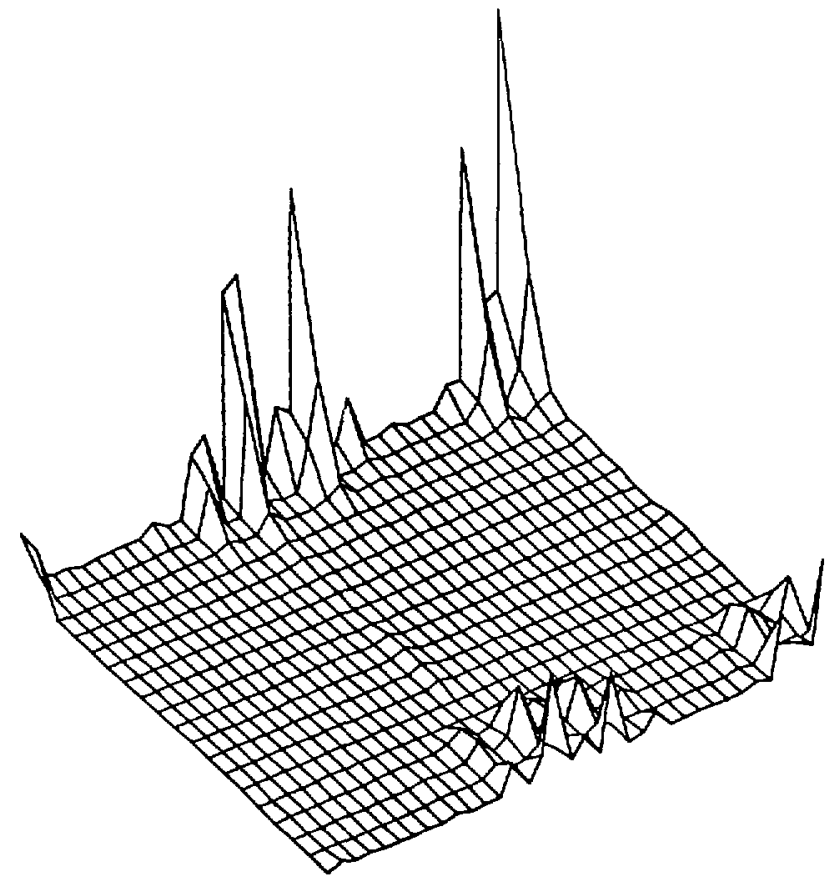

Fig. 7. Excitation 2. Higher threshold for inverting $\Sigma$.

parameters (masses and stiffness parameters). This point is currently under investigation.

Finally, it is of interest to notice that, because of our approach for change detection and diagnosis, we have also addressed in this paper the problem of optimal sensor location for parameter identification. Actually, we show in [12] that the inverse of the matrix $\Gamma$ in (20), which characterizes the asymptotic power of the instrumental test (19), is equal to the asymptotic covariance matrix of the estimation error of the optimal instrumental variable identification method. As we have addressed the optimal sensor location problem using as a criterion the power of the instrumental 


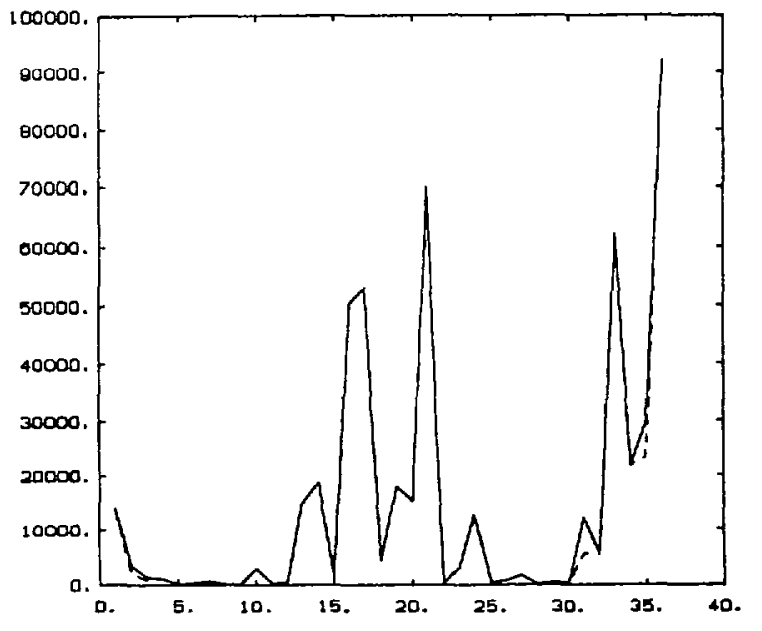

$-\lambda=0.99$

$-\cdots \lambda=0.999$

Fig. 8. Excitation 2. Global criterions for two values of $\lambda$.

test, we also solved the problem of optimal sensor location for parameter identification.

\section{REFERENCES}

[1] A. Arbel, "Sensor placement in optimal filtering and smoothing problems," IEEE Trans. Automat. Contr., vol. AC-27, pp. 94-98, Feb. 1982.

[2] M. Basseville, A. Benveniste, and G. Moustakides, "Detection and diagnosis of abrupt changes in modal characteristics of nonstationary digital signals," IEEE Trans. Inform. Theory, vol. IT-32, pp. 412417, May 1986.

[3] M. Basseville, A. Benveniste, G. Moustakides, and A. Rougée, "Detection and diagnosis of changes in the eigenstructure of nonstationary multivariable systems," Automatica, vol. 23, pp. 479-489, July 1987.

[4] C. D. Johnson, "Optimization of a certain quality of complete controllability and observability for linear dynamical systems," Trans. ASME, Ser. D, vol. 91, pp. 228-238, 1969.

[5] R. L. Kosut, A. Arbel, and K. M. Kessler, "Optimal sensor system design for state reconstruction," IEEE Trans. Automat. Contr., vol. AC-27, pp. 242-244, Feb. 1982.

[6] C. S. Kubrusly and H. Malebranche "Sensors and controllers location in distributed systems. A survey," Automatica, vol. 21, pp. 117-128, Mar. 1985.

[7] R. K. Mehra, "Optimization of measurement schedules and sensor design for linear dynamic systems," IEEE Trans. Automat. Contr., vol. AC-21, pp. 55-64, Feb. 1976.

[8] P. C. Müller and H. I. Weber, "Analysis and optimization of certain qualities of controllability and observability for linear dynamical systems," Automatica, vol. 8, pp. 237-246, 1972.

[9] A. Munak, "Optimal sensor location for identification of unknown parameters in a bubble-column loop bireactor," in Proc. 6th Intern. Conf. on "Analysis and Optimization of Systems," Nice, France, June 1984, LNCIS 62-63, pp. 415-433.

[10] S. Omatu, S. Koide, and T. Soeda, "Optimal sensor location problem for a linear distributed parameter system," IEEE Trans. Automat. Contr., vol. AC-23, pp. 665-673, Aug. 1978.
[11] A. Rougée, "Détection de changements dans les paramètres AR d'un processus ARMA vectoriel: Application à la surveillance des vibrations" (in French), Thèse 3ème Cycle, Univ. de Rennes I, France, Sept. 1985.

[12] A. Rougée, M. Basseville, A. Benveniste, and G. Moustakides, "Optimum robust detection of changes in the AR part of a multivariable ARMA process," IEEE Trans. Automat. Contr., vol. AC-32, Sept. 1987.

[13] F. E. Udwadia and J. A. Garba, "Optimal sensor locations for structural identification," in Proc. NASA and JPL Workshop on "Identification and Control of Flexible Space Structures," San Diego, CA, July 1984, Apr. 1985, pp. 247-261.

[14] W. E. Vander Velde and C. R. Carignan, "Number and placement of control system components considering possible failures," $J$. Guidance, Contr. Dynam., vol. 7, pp. 703-709, Noy.-Dec. 1984

[15] K. Watanabe, M. Sasaki, and D. M. Himmelblau, "Determination of optimal measuring sites for fault detection of non-linear systems," Int. J. Syst. Sci., vol. 16, pp. 1345-1364, Nov. 1985.

[16] J. N. Juang and G. Rodriguez, "Formulations and applications of large structure actuator and sensor placement," presented at the 2nd VPI\&SU/AIAA Symp. Dynam. Contr. Large Flexible Spacecraft, Blacksburg, VA, June 1979

[17] P. C. Hughes and R. E. Skelton, "Controllability and observability for flexible spacecraft,"' J. Guidance Contr., vol. 3, pp. 452-459, Sept.Oct. 1980

[18] T. Li and R. W. Longman, "Comparison and generation of alternative degree of controllability and degree of observability concepts," presented at the AIAA/AAS Astrodynamics Specialist Conf., San Diego, CA, Aug. 1982.

Michèle Basseville, for a photograph and biography, see p. 592 of the July 1987 issue of this TRANSACTIONS.

Albert Benveniste (M'81), for a photograph and biography, see p. 591 of the July 1987 issue of this TRANSACTIONS.

Georges B. Moustakides (''79-M'82-SM'82-M'83), for a photograph and biography, see p. 592 of the July 1987 issue of this TRANSACTIONS.

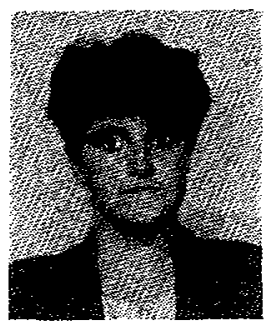

Anne Rougée was born in France in 1959. She received the Diplôme d'Etudes Approfondies in Statistics from Université de Paris XI, Orsay, France, in 1983, and the Thèse de Troisième Cycle from the Université de Rennes, France, in 1985.

She attended the Department of Mathematics at the Ecole Normale Superieure de l'Enseignement Technique, Cachan, France, from 1979 to 1983, and was a Research Assistant at the Institut de Recherche en Informatique et Systèmes Aléatoires, Rennes, France, from 1983 to 1985 . Afterwards, she spent one year working at the Laboratory for Information and Decision Systems, Massachusetts Institute of Technology, Cambridge, as a Visiting Scientist supported by the Institut National de la Recherche en Informatique et Automatique, Le Chesnay, France. Since 1986 she has been a Research Engineer at Thomson-CGR, Buc, France. Her main areas of interest are estimation, detection, computer vision, and medical image processing. 